\title{
Development of a Facile Fluorescent Assay for the Detection of 80 Mutations Within the p53 Gene
}

\author{
Darren R. Hodgson, ${ }^{1}$ Carole A. Foy, ${ }^{1}$ Maxine Partridge, ${ }^{2}$ Stelios Pateromichelakis, ${ }^{2}$ and \\ Neil J. Gibson ${ }^{1}$ \\ ${ }^{1}$ AstraZeneca Diagnostics, Cheshire, United Kingdom \\ ${ }^{2}$ Maxillofacial Unit/Oncology, King's College Hospital, Denmark Hill, London, United Kingdom \\ Accepted March 3, 2002
}

\begin{abstract}
Background: Alterations in the p53 tumor suppressor gene constitute one of the most frequent genetic events associated with the development of human cancers. Determination of an individual's p53 status may be of value in early diagnosis, prediction of response to treatment, and for the detection of minimal residual cancer. Recent studies have also revealed that specific mutations affecting the p53 gene are associated with a poor outcome. The majority of tumor biopsies that are sent for study in the laboratory contain neoplastic cells intermingled with stroma, such that the detection of alterations in the p53 gene requires a tumor enrichment technique and/or highly sensitive mutation detection technologies. Thus, it is desirable that a clinically useful assay for detecting point mutations in the p53 gene function in the presence of significant
\end{abstract}

quantities of wild-type sequence and identify the critical sequence aberrations.

Materials and Methods: We utilized molecular beacons in a real-time allele-specific PCR format to obtain reference data on samples of quantitatively known p53 mutation status. These data have been statistically analyzed and the results used to detect p53 mutations, indicating the presence of occult tumor.

Results: We describe validation of a simple, rapid, sensitive, and quantitative ARMS assay for identifying the levels of 80 point mutations within the p53 gene that, when mutated, constitute at least $1 \%$ of the total p53 sequences. Conclusions: The assay successfully identifies rare p53 gene mutations in clinical samples and overcomes many of the limitations of current technologies.

\section{Introduction}

Mutation of the p53 tumor suppressor gene is one of the most frequently reported events in neoplastic cells, occurring in approximately $50 \%$ of human cancers (1). There have been many reports concerning the utility of p53 gene alterations for tumor diagnosis $(2,3)$ and management of cancer patients (4-8). Such studies have come to the fore as our understanding of the pathways involved in apoptotic cell death and the response to DNA damage has increased. Tumors harboring a subset of mutations in the conserved core region, encoded by exons 5-8, have been associated with reduced survival or poor response to therapy, when compared to mutations that lie outside of this region, or wild-type sequence (7-16).

However, results from studies evaluating specific tumor types, for example squamous tumors in the head and neck region, often conflict with reports indicating that $\mathrm{p} 53$ mutations are associated with sensitivity to chemotherapy or radiotherapy (16-22). It is important to recognize that these differences reflect the fact that it is impossible to separate sensitivity to a

Correspondence and reprint requests should be addressed to: Neil Gibson, AstraZeneca Diagnostics, Gadbrook Park, Northwich, Cheshire, CW9 7RA, United Kingdom. Phone: (0) 1625 519714; fax: +44 (0) 1625 514463; e-mail neil.gibson@astrazeneca.com. given treatment from the overall biological behavior of a tumor (23). Thus, unraveling the relationship between genetic aberrations and response to treatment will require in-depth study of the genetic background and clinic pathologic features before substantive conclusions can be reached.

Mutations affecting the p53 gene have been shown to have diagnostic value for patients with squamous tumors of the head and neck. Recent studies, using a molecular diagnostic tool based on finding the same p53 gene mutation in a primary tumor and the surrounding normal tissue, pronounced tumor-free after conventional light microscopy, detected occult tumor in approximately one-third of all patients tested $(2,3,24)$. In our latest report, molecular evidence of residual cancer was round in both mucosal and deep muscle surgical margins (3). At present, the significance of finding a p 53 gene mutation in mucosal margins is unclear, but the presence of this aberration in muscle biopsies clearly identifies residual tumor, which is frequently responsible for the patient's demise. Based on these findings, a multinational study has begun to establish whether the molecular diagnostic identifies more cases with residual disease than conventional light microscopy, since detection of sub-microscopic residual disease at an early stage would enable clinicians to ensure that these patients are targeted to receive adjuvant 
treatment at an early stage, when it would be most cost-effective and reduce morbidity and mortality.

At present, the results obtained using the molecular test are not taken into account when planning adjuvant treatment, typically postoperative radiotherapy, and a proportion of cases with occult p53 mutation-positive residual tumor develop a recurrence at the operative site, despite receiving full-dose adjuvant treatment. Whether this treatment fails because these patients have tumors which are clinically aggressive, or because they lack functional p53, or harbor other genetic abnormalities which modulate radiosensitivity, is not clear at present. However, this observation highlights the need for prospective studies to investigate the molecular determinants of response to adjuvant treatments, and also to develop novel approaches to deal with the problem of radioresistant minimal residual cancer (25).

Current approaches to screen for p53 mutations include immunohistochemistry (IHC) (26), a functional assay of separated alleles in yeast (FASAY) (27) and genetic analyses such as enzymatic or hybridization sequencing, and single-strand conformation polymorphism analysis (SSCP) $(28,29,30)$. The current experiment, to establish the prognostic significance of molecular detection of tumor-positive surgical margins, utilizes FASAY and a p 53 phage-plaque assay. In essence, the techniques involve screening p53 cDNA for the presence of a p53 mutation by cloning into an indicator yeast strain, sequencing cDNA to identify the nature of the p 53 aberration, and then designing a mutant-specific oligonucleotide probe to detect the presence of the same mutation in phage libraries prepared from surgical margins which are pronounced tumor-free by the histopathologist. Although highly sensitive, this methodology is extremely time consuming and the result is not available for 4-6 weeks. The assay often needs to be repeated to ensure sensitivity and specificity in cases where only low levels of mutant are detected. Critical decisions about the need for adjuvant treatment need to be made at an early stage; it is well-recognized that early repopulation of the tumor at the operative site contributes to local relapse (31). Any assay must be sufficiently sensitive to directly identify mutations in the presence of significant quantities of normal tissue, preferably incorporate closed tube technology to avoid cross-contamination of patient samples, be automatable to increase sample throughput, and be sufficiently rapid to provide quantitative data to aid postoperative patient management.

We developed a panel of sensitive assays for specific $\mathrm{p} 53$ mutations using real-time allele-specific amplification (ARMS). ARMS is a powerful technique for detecting mutations and polymorphisms in DNA (32). Primers are designed to match the mutant sequence at their $3^{\prime}$ termini. Facile amplification results from a mutant template and significantly inhibited amplification from a 3' mismatched, wild-type sequence. In a real-time PCR assay, the production of PCR product results in a fluorescent signal that is monitored throughout PCR. The threshold cycle $(\mathrm{Ct})$ - the point at which signal becomes distinguishable from background noiseindicates the input copy number of target sequence (33). In a real-time ARMS reaction, the Ct is also affected by the proportion of the sample matched to the $3^{\prime}$ end of the primer. Therefore, by performing both a PCR and an ARMS reaction on a sample, the proportion of mutant sequence present can be quantified. For any given control $\mathrm{Ct}$ (indicating total copy number) the proportion of mutant sequences can be determined by comparing the ARMS Ct for the sample with reference data from samples of known composition.

In the present study, we employed a molecular beacons format (34) to generate sequence-specific signals during thermal cycling. Molecular beacons are single-strand nucleic acids composed of selfcomplementary sequences flanking a central region. The central region is designed to be complementary to sequences anticipated to be produced on amplification. In the absence of specific amplification product, the flanking regions remain self-hybridized and hold a terminal fluorophore and quencher molecule in close proximity. In the presence of the amplification, product a more stable interaction between it and the central region of the beacon predominates and physically separates fluorophore and quencher. The reduction in fluorescent resonance energy transfer results in an increase in fluorescent signal. We used this methodology to produce an assay for the 80 mutations occurring in the p53 gene. The assay covers $42 \%$ of reported mutations, insertions, and deletions in head and neck cancer (see Table 1 and [35]). We describe the production of the assay and the acquisition and analysis of reference data that enable the rapid, quantitative, and sensitive detection of specific p53 mutations in clinical samples. We demonstrate the use of the assay for detecting minimal residual cancer in biopsies of normal tissue following surgical ablation of squamous tumors of the head and neck. The assay may be of widespread utility as a sensitive marker of cancer cells and for patient stratification based on the functional consequences of the p53 mutation detected.

\section{Materials and Methods}

\section{Instrumentation}

PCR reactions without real-time monitoring were carried out on a PE 480 thermal cycler (Applied Biosystems, Foster City, CA, USA). PCR reactions with real-time fluorescent monitoring were carried out in optical thermal cycling plates on the ABI prism 7700 thermal cycler (Applied Biosystems).

\section{Reagents and Solutions}

DNA size marker (50 bp) and dNTPs were from Pharmacia Biotechnology (Piscataway, NJ, USA), DNA polymerase (AmpliTaq Gold) from P.E. Applied 
Table 1. Sequences of ARMS primers used to detect 80 mutations in the p53 gene

\begin{tabular}{|c|c|c|c|c|c|}
\hline Well & Codon & Change & Frequency & ARMS Primer (5' to $\left.3^{\prime}\right)$ & $\begin{array}{l}\text { Reverse PCR Primer/ } \\
\text { Molecular Beacon }\end{array}$ \\
\hline 3 & 132 & AAG to AGG & 0.357 & ggt ctt gac cag ttg gca aaa cat tc & $861-98 / 5 a U$ \\
\hline 4 & 132 & AAG to $\mathrm{CAG}$ & 0.071 & tcc tac agt act ccc ctg ccc tca tcc & $862-98 / 6 a L$ \\
\hline 5 & 135 & TGC to TAC & 0.071 & gta ctc ccc tge cct cac caa gat gtt gta & $862-98 / 6 a L$ \\
\hline 6 & 141 & TGC to TAC & 0.143 & aac aag atg ttt tgc caa ctg gcc aag aca ta & $862-98 / 6 a L$ \\
\hline 7 & 151 & CCC to TCC & 0.214 & tgg cgt gga tgc ggg tgc cgg gcg gag a & $861-98 / 5 a U$ \\
\hline 8 & 151 & CCC to ACC & 0 & cct gtg cag ctg tcg gtt gat tcc ata a & $862-98 / 6 a L$ \\
\hline 9 & 152 & CCG to CTG & 0.143 & gca gct gtg ggt tga ttc cac acc tct & $862-98 / 6 a \mathrm{~L}$ \\
\hline 10 & 156 & CGC to CCC & 0.071 & cca cac ccc cge ccg gca ctc c & $862-98 / 6 a \mathrm{~L}$ \\
\hline 11 & 157 & GTC to TTC & 0.929 & aca ccc ccg ccc ggc acc ctc $t$ & $862-98 / 6 a \mathrm{~L}$ \\
\hline 12 & 158 & CGC to CTC & 0.286 & cac acc ccc tcc cgg cac ccg cgt gct & $862-98 / 6 a \mathrm{~L}$ \\
\hline 15 & 158 & CGC to CAC & 0.571 & gat tcc aca ccc ccg ccc ggc acc ctc gta ca & $862-98 / 6 a L$ \\
\hline 16 & 161 & GCC to ACC & 0.143 & gcc agg cac ccg cgt ccg cgc cag ga & $862-98 / 6 a L$ \\
\hline 17 & 163 & TAC to TGC & 0.429 & tcc gtc atg tgc tgt gac tgc tgg $c$ & $861-98 / 5 a U$ \\
\hline 18 & 173 & GTG to ATG & 0.143 & cta caa gca gtc aca gca cat gac gga gga ta & $862-98 / 6 a L$ \\
\hline 19 & 173 & GTG to TTG & 0.357 & aca agc agt cac agc aca tgc cgg agg att & $862-98 / 6 a L$ \\
\hline 20 & 175 & CGC to CAC & 5.071 & cac agc aca tga cgg agg ttg tga ggg a & $862-98 / 6 a L$ \\
\hline 21 & 175 & CGC to AGC & 0 & cac agc aca tga cgg agg ttg tga cga & $862-98 / 6 a L$ \\
\hline 22 & 175 & CGC to TGC & 0.071 & cac agc aca tga cgg agg ttg tga agt & $862-98 / 6 a L$ \\
\hline 23 & 175 & CGC to CCC & 0 & aca gca cat gac gga ggt tgt gag ccc & $862-98 / 6 a L$ \\
\hline 24 & 175 & CGC to CTC & 0 & aca gca cat gac gga ggt tgt gag tct & $862-98 / 6 a L$ \\
\hline 27 & 176 & TGC to TAC & 0.286 & cat gac gga ggt tgt gag gcg gta & $862-98 / 6 a \mathrm{~L}$ \\
\hline 28 & 176 & TGC to TTC & 4.214 & gca cat gac gga ggt tgt gag gcg gtt & $862-98 / 6 a L$ \\
\hline 29 & 177 & CCC to CGC & 0 & cat gac gga ggt tgt gag gcg ctg ctg & $862-98 / 6 a L$ \\
\hline 30 & 179 & CAT to CGT & 0.429 & agg ttg tga ggc gct gcc ccc act g & $862-98 / 6 a L$ \\
\hline 31 & 179 & CAT to TAT & 0.500 & tga cgg agg ttg tga ggc gct gcc ccc att & $899-97 / 6 a L$ \\
\hline 32 & 192 & CAG to TAG & 0.214 & gat tgc tct tag gtc tgg ccc ctc ttt & 694-98/6bL \\
\hline 33 & 193 & CAT to CGT & 0.286 & gct ctt agg tct ggc ccc tcc tca ccg & 694-98/6bL \\
\hline 34 & 195 & ATC to ACC & 0.500 & ctt agg tct ggc ccc tcc tca gca tct gac & 694-98/6bL \\
\hline 35 & 196 & CGA to TGA & 1.000 & ata ctc cac acg caa att tcc ttc cac aca & $861-98 / 5 a U$ \\
\hline 36 & 205 & TAT to TGT & 1.000 & cga aaa gtg ttt ctg tca tcc aat c & $863-98 / 6 a L$ \\
\hline 39 & 213 & CGA to TGA & 0.786 & gga gta ttt gga tga cag aaa cac ttc tt & 694-98/6bL \\
\hline 40 & 220 & TAT to TGT & 1.571 & aca tag tgt ggt ggt gcc gtg & 694-98/6bL \\
\hline 41 & 228 & GAC to AAC & 0 & gcc tgt gtt atc tcc tag gtt ggc tgt a & $644-98 / 7 a \mathrm{~L}$ \\
\hline 42 & 234 & TAC to TGC & 0.714 & ccc atg cag gaa ctg tta cac atg tag atc c & $643-98 / 7 b U$ \\
\hline 43 & 237 & ATG to ATA & 0.571 & gac tgt acc acc atc cac tac aac tac aaa & 644-98/7aL \\
\hline 44 & 238 & TGT to TAT & 0.214 & ctg tac cac cat cca cta caa cta cat cta & 644-98/7aL \\
\hline 45 & 241 & TCC to TTC & 0.214 & tcc agt tca tgc cgc cca tgc aca & $643-98 / 7 b U$ \\
\hline 46 & 242 & TGC to TTC & 0.500 & acc atc cac tac aac tac atg tgt aac agt tct tt & $644-98 / 7 \mathrm{aL}$ \\
\hline 47 & 244 & GGC to TGC & 0.214 & cta caa cta cat gtg taa cag ttc ctg cat ct & 644-98/7aL \\
\hline 48 & 245 & GGC to AGC & 1.714 & tac gtg tgt aac agt tcc tgc atg gtc a & 644-98/7aL \\
\hline 51 & 245 & GGC to GAC & 0.786 & cat gtg taa cag ttc ctg cat ggg cta & 644-98/7aL \\
\hline 52 & 245 & GGC to TGC & 0.429 & act aca tgt gta aca gtt cct gca tgg tct & 644-98/7aL \\
\hline 53 & 245 & GGC to GTC & 0.214 & cat gtg taa cag ttc ctg cat ggg agt & 644-98/7aL \\
\hline 54 & 245 & GGC to CGC & 0 & aca tgt gta aca gtt cct gca tgg ccc & 644-98/7aL \\
\hline
\end{tabular}


Table 1. (Continued)

\begin{tabular}{|c|c|c|c|c|c|}
\hline Well & Codon & Change & Frequency & ARMS Primer $\left(5^{\prime}\right.$ to $\left.3^{\prime}\right)$ & $\begin{array}{c}\text { Reverse PCR Primer/ } \\
\text { Molecular Beacon }\end{array}$ \\
\hline 55 & 245 & GGC to GCC & 0 & cat gtg taa cag ttc ctg cat ggg agc & $644-98 / 7 \mathrm{aL}$ \\
\hline 56 & 248 & CGG to $C A G$ & 2.571 & aca gtt cct gca tgg gcg gca tga aac a & $644-98 / 7 a L$ \\
\hline 57 & 248 & CGG to TGG & 2.071 & aac agt tcc tgc atg ggc ggc atg aag t & $644-98 / 7 \mathrm{aL}$ \\
\hline 58 & 248 & CGG to CTG & 0.357 & taa cag ttc ctg cat ggg cgg tat gaa tct & $644-98 / 7 a \mathrm{~L}$ \\
\hline 59 & 248 & CGG to GGG & 0.143 & gta aca gtt cct gca tgg gcg gcg tga agg & $644-98 / 7 \mathrm{aL}$ \\
\hline 60 & 248 & CGG to CCG & 0.071 & tgt aac agt tcc tgc atg ggc ggc atg aac ac & $644-98 / 7 a \mathrm{~L}$ \\
\hline 63 & 249 & AGG to AGT & 0.357 & ttc ctg cat ggg cag cat gaa ccg gtg t & $644-98 / 7 a \mathrm{~L}$ \\
\hline 64 & 249 & AGG to ATG & 0 & ttc ctg cat ggg cga cat gaa ccg tat & $644-98 / 7 a \mathrm{~L}$ \\
\hline 65 & 249 & AGG to AGC & 0 & gca tgg gcg gca tga acc gga ac & $644-98 / 7 a \mathrm{~L}$ \\
\hline 66 & 249 & AGG to GGG & 0.286 & ttc gag tgt gat gat ggt gag gat ggg cgc & $643-98 / 7 b U$ \\
\hline 67 & 249 & AGG to TGG & 0.214 & ttg cag tgt gat gat ggt gag gat ggg aca & $643-98 / 7 b U$ \\
\hline 68 & 249 & AGG to ACG & 0.071 & aac agt tcc tgc atg ggc gac atg aac cgg tc & 644-98/7aL \\
\hline 69 & 249 & AGG to AAG & 0 & atg ggc agc atg aac cga aa & 644-98/7aL \\
\hline 70 & 258 & GAA to AAA & 0 & ggc cca tcc tca cca tca tca cac tta & 644-98/7aL \\
\hline 71 & 258 & GAA to GGA & 0.071 & cca tcc tca cca tca tca cac tgc g & 644-98/7aL \\
\hline 72 & 266 & GGA to GTA & 0 & tcc tat cct gag tag tgg taa tct act tgt & $629-98 / 8 \mathrm{aL}$ \\
\hline 75 & 266 & GGA to AGA & 0.286 & ttc cta tcc tga gta gtg gta atc tac gga & $629-98 / 8 \mathrm{aL}$ \\
\hline 76 & 266 & GGA to GAA & 0.214 & tcc tat cct gag tag tgg taa tct act gaa & $629-98 / 8 \mathrm{aL}$ \\
\hline 77 & 272 & GTG to ATG & 0 & atc tac tgg gac gga aca gct ttg tga & $629-98 / 8 \mathrm{aL}$ \\
\hline 78 & 273 & CGT to CAT & 2.429 & ggg acg gaa cag ctt tga ggt gaa & $629-98 / 8 \mathrm{aL}$ \\
\hline 79 & 273 & CGT to TGT & 1.143 & act ggg acg gaa cag ctt tga ggt ct & $629-98 / 8 \mathrm{aL}$ \\
\hline 80 & 273 & CGT to CTT & 0.714 & tac tgg gac gga aca gct ttg agg tac $t$ & $629-98 / 8 \mathrm{aL}$ \\
\hline 81 & 273 & CGT to AGT & 0 & tac tgg gac gga aca gct ttg agg aga & $629-98 / 8 \mathrm{aL}$ \\
\hline 82 & 273 & CGT to GGT & 0.143 & tac tgg gac gga aca gct ttg agg agg & $629-98 / 8 \mathrm{aL}$ \\
\hline 83 & 273 & CGT to CCT & 0.071 & act ggg acg gaa cag ctt tga ggt gac & $629-98 / 8 \mathrm{aL}$ \\
\hline 84 & 275 & TGT to TAT & 0.357 & acg gaa cag ctt tga ggt gcg tgt tga & $629-98 / 8 \mathrm{aL}$ \\
\hline 87 & 278 & CCT to CTT & 0.214 & aac agc ttt gag gtg cgt gtt tgt gcc tgt gt & $629-98 / 8 \mathrm{aL}$ \\
\hline 88 & 278 & CCT to TCT & 0.786 & gaa cag ctt tga ggt gcg tgt ttg tgc cta tt & $629-98 / 8 \mathrm{aL}$ \\
\hline 89 & 280 & AGA to ACA & 0.071 & ggt gcg tgt ttg tgc ctg tcc tgg aac & $629-98 / 8 \mathrm{aL}$ \\
\hline 90 & 282 & CGG to TGG & 1.643 & tgc gtg ttt gtg cct gtc ctg gga gag agt & $629-98 / 8 \mathrm{aL}$ \\
\hline 91 & 282 & CGG to CCG & 0 & gtg cgt gtt tgt gcc tgt cct ggg aga gac gc & $629-98 / 8 \mathrm{aL}$ \\
\hline 92 & 282 & CGG to GGG & 0.071 & ggt gcg tgt ttg tgc ctg tcc tgg gag agt cg & $629-98 / 8 \mathrm{aL}$ \\
\hline 93 & 282 & CGG to CAG & 0 & gtg cgt gtt tgt gcc tgt cct ggg aga gac ga & $629-98 / 8 \mathrm{aL}$ \\
\hline 94 & 285 & GAG to AAG & 0.571 & gcc tgt cct ggg aga gac cgg cgc aga a & $629-98 / 8 \mathrm{aL}$ \\
\hline 95 & 286 & GAA to AAA & 0.214 & tcc tgg gag aga ccg gcg cac agc ga & $629-98 / 8 \mathrm{aL}$ \\
\hline 96 & 298 & GAG to TAG & 1.000 & ccg caa gaa agg gga gcc tca cca gt & 629-98/8aL \\
\hline
\end{tabular}

Key to legend:

Well $=$ position on assay plate,

codon $=$ p5 3 codon

change $=$ mutation in $\mathrm{p} 53$ gene sequence

Frequency $=$ frequency of mutation occurance in p53 database [34] as a percentage of all mutations reported for head and neck cancer (excluding data from cell lines)

ARMS primer $=$ primer sequence used to detect mutation

Reverse PCR Primer/Molecular beacon = reverse PCR primer used with ARMS primer and molecular beacon used to detect PCR amplification (see table 3 for sequences). 
Biosystems, NuSieve Agarose from FMC Bioproducts (Rockland, MD, USA), and ?X174 RF DNA HaeIII digest molecular size marker from Life Technologies. Spin columns for PCR product purification were from Qiagen (Hilden, Germany). All other chemicals were from Aldrich Chemical Company Co. (Milwaukee WI). Molecular beacons were prepared by Oswel Research Products (Southampton UK). The remaining oligonucleotides were synthesized on a P.E. Applied Biosystems 394 DNA/RNA synthesizer with P.E. Applied Biosystems reagents according to the manufacturers protocols and were purified by size exclusion filtration through NAP10 columns (Pharmacia). ARMS buffer consists of $1.2 \mathrm{mM} \mathrm{MgCl} 2,10 \mathrm{mM}$ Tris- $\mathrm{HCl}, 50 \mathrm{mM} \mathrm{KCl}, \mathrm{pH}$ 8.3, and cassette dilution buffer consisting of 10 $\mathrm{mM}$ Tris- $\mathrm{HCl}, 50 \mathrm{mM} \mathrm{KCl}$, and $1 \mathrm{~g} / \mathrm{L} \mathrm{BSA}, \mathrm{pH} \mathrm{8.3.}$

\section{Samples}

Ethical Committee Approval for the minimal residual cancer study was granted at King's College Hospital. Biopsies of primary oral SCCs were harvested at the time of primary surgery and stored at $-70^{\circ} \mathrm{C}$ until required. Following excision of the primary tumor, four mucosal and two deep surgical margins, each approximately $1 \mathrm{~cm} \times 8 \mathrm{~mm} \times 8 \mathrm{~mm}$ were harvested. Each margin was divided into two; the inner portion was processed for conventional light microscopy, the outer half frozen for molecular analysis. Cases reported as having tumor-positive margins by the histopathologist were not forwarded for molecular analysis. All samples were coded and analyzed blind.

\section{DNA Preparation}

DNA was prepared from tumor frozen portion of the surgical margins using DNA STAT 50 (Biogenesis, Poole, UK) according to the manufacturer's instructions.

\section{Template Production for Real-Time Analyses}

A multiplex PCR was performed on neat and 1 in 1000 diluted DNA samples derived from peripheral blood or tumors to amplify 477-, 236-, and 415-bp fragments incorporating p53 exons $(5,6), 7$ and 8,9, respectively. The following primers were used in combination at the final concentrations shown; 5' tga ctt tca act ctg tct cct tc (419 nM), 5' acc cgg agg gcc act gac aac (419 nM), 5' cac agg tct ccc caa ggc gca c (93 nM), 5' ggg ccc agg ggt cag cgg caa (93 nM), 5' ttc ctt act gcc tct tgc ttc tct (488 nM), and 5' aag aag aaa acg gca ttt tga gtg (488 $\mathrm{nM})$. The PCR mix also contained dNTPs (100 mM), Taq DNA polymerase (1 unit), and the appropriate DNA template $(2.5 \mu \mathrm{l})$ in ARMS buffer (total volume $25 \mu \mathrm{l}$ ). Reactions were cycled $1 \times$ $\left(94^{\circ} \mathrm{C}\right.$ for $\left.20 \mathrm{~min}\right), 40 \times\left(94^{\circ} \mathrm{C}\right.$ for $1 \mathrm{~min}, 60^{\circ} \mathrm{C}$ for $1 \mathrm{~min}), 1 \times\left(72^{\circ} \mathrm{C}\right.$ for $\left.10 \mathrm{~min}\right)$. Mutant p53 fragments or cassettes were prepared by the method of Higuchi (37) (Fig.1). The cassettes were examined by agarose gel electrophoresis to a) check that a single fragment of the correct size had been prepared and b) permit approximate quantitation of the cassette

1st Round. Synthesis of mutant half cassettes in two tubes
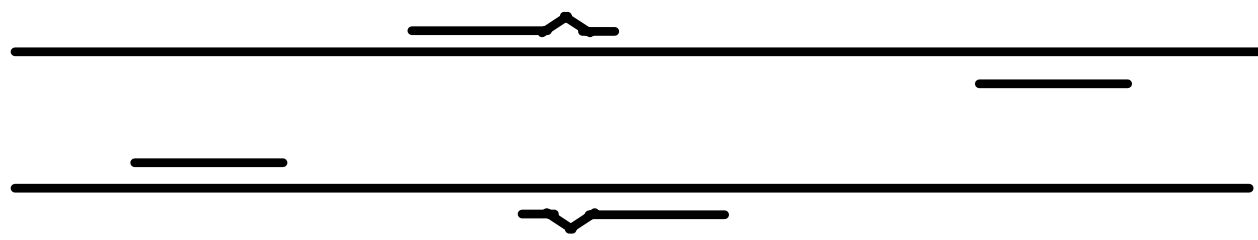

2nd Round. Megapriming of half cassettes to produce full length mutated template

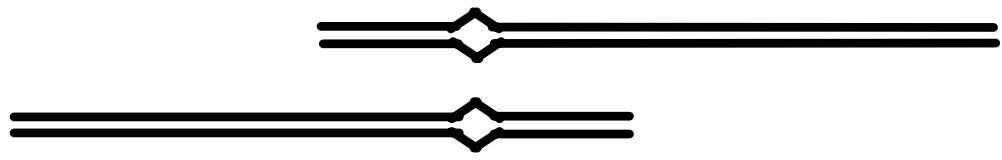

2nd Round. Nested primers produce final cassette

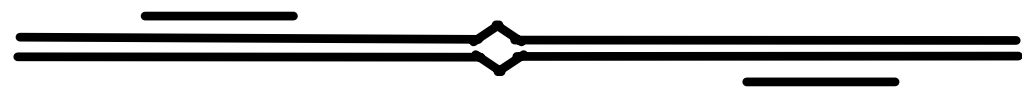

Final mutated cassette

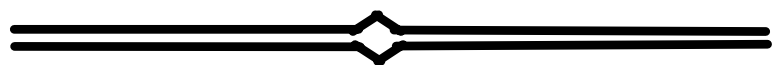

Fig. 1. Preparation of synthetic mutated templates. The method is based on the method of Higuchi et al. (37). In the first round, two fragments are created in separate reactions, each harboring the primer-induced mutation. In the second round, overlapping strands from the two fragments hybridize and produce a cassette with the mutation in the center. Nested primers, also in the second round, then amplify the desired product. 
Table 2. Sequences of control primers used in the p53 assay

\begin{tabular}{cccc}
\hline Wells & Exon & Primer (5' to 3') & $\begin{array}{c}\text { Reverse PCR Primer/ } \\
\text { Molecular Beacon }\end{array}$ \\
\hline $1,2,13,14$ & 6 & ccc agg gtc ccc agg cct ctg att & $862-98 / 6 a \mathrm{~L}$ \\
$25,26,37,38$ & 6 & ccc agg gtc ccc agg cct ctg att & $694-98 / 6 \mathrm{bL}$ \\
$49,50,61,62$ & 7 & cac agg tct ccc caa ggc gca c & $644-98 / 7 \mathrm{aL}$ \\
$73,74,85,86$ & 8 & ttc ctt act gcc tct tgc ttc tct & $629-98 / 8 \mathrm{aL}$ \\
\hline
\end{tabular}

Key to legend:

Well $=$ position on assay plate,

exon $=$ p53 exon

Primer $=$ Forward primer sequence used to perform control reaction

Reverse PCR Primer/Molecular beacon = reverse PCR primer used with forward primer and molecular beacon used to detect PCR amplification (see table 3 for sequences).

by comparing the band intensities with those of the ?X174 RF DNA HaeIII digest, which contains known amounts of DNA. Cassettes were diluted to a working concentration in cassette dilution buffer and admixtures of mutant cassettes in a wild-type p 53 multiplex product background were created by mixing samples with equivalent $\mathrm{Cts}$ in control real-time PCRs.

\section{Design of ARMS Primer and Molecular Beacons}

ARMS and reverse primers (Tables 1 and 3) were designed to detect the 80 prevalent mutations (excluding cell lines) of human p53 in the database maintained by T. Soussi (35). Each ARMS reaction could be correlated with a further mutation independent control PCR (Tables 2 and 3), which utilized the same multiplex product as template. The ARMS primers incorporate additional mismatches at position -2 or -3 . Molecular beacons (34) were designed to anneal to sequences between amplification primer sites. The open and closed forms of the beacons were modeled for stability on the website of Michael Zuker (38). Beacons generally comprised 5-mer self-complementary hairpins and 24-27 base central probe regions.

\section{Real-Time Amplification Conditions}

Each of the 96 wells on the optical plates contained a reverse and a control or ARMS primer at $500 \mathrm{nM}$ and a molecular beacon at $400 \mathrm{nM}(800 \mathrm{nM}$ for beacon 6bL) (see Tables 1 and 2 for plate configuration). In addition, each well contained $100 \mathrm{nM}$ dNTPs, 60 nM Rox passive reference (Perkin Elmer, Warrington, UK), I× ARMS buffer, I U of AmpliTaq Gold, and DNA template in a total volume of $25 \mu \mathrm{l}$. For use on unknown samples, plates containing a $2 \times$ concentrate of well-specific reagents were stored frozen and a mixture of template and common reagents added by multipipette or immediately prior to use. Plates were cycled as follows: $1 \times\left(94^{\circ} \mathrm{C}\right.$ for $\left.20 \mathrm{~min}\right), 50 \times\left(94^{\circ} \mathrm{C}\right.$ for $45 \mathrm{sec}, 60^{\circ} \mathrm{C}$ for $45 \mathrm{sec}$ ). Rox on and quencher off were selected and FAM signals were collected during the $60^{\circ} \mathrm{C}$-annealing phase.

\section{Preparation of 80 Reference Data Sets}

Data points were collected, upon independent runs on two separate 7700 machines, for each of the 80 ARMS and 4 control reactions on dilutions of samples of known composition. Wild-type multiplex templates from 35 individuals were used. Mutant data, a minimum of nine independent data points, were generally

Table 3. Sequences of reverse primers and molecular beacons

\begin{tabular}{|c|c|}
\hline ID & Sequence $\left(5^{\prime}\right.$ to $\left.3^{\prime}\right)$ \\
\hline $629-98$ & gaa tct gag gca taa ctg cac cct $t$ \\
\hline $643-98$ & cac agg tct ccc caa ggc gca c \\
\hline $644-98$ & agc ggc aag cag agg ctg ggg $\mathrm{c}$ \\
\hline $861-98$ & tga ctt tca act ctg tct cct tc \\
\hline $862-98$ & tct gtc atc caa ata ctc cac acg caa \\
\hline $694-98$ & acc cgg agg gcc act gac aac \\
\hline $5 a U$ & ggc cgc tct tcc tac agt act ccc ctg ccc ggc $\mathbf{c}$ \\
\hline $6 \mathrm{aL}$ & cgc ccg gat aag atg ctg agg agg ggc cag ggc $\mathbf{g}$ \\
\hline $6 \mathrm{bL}$ & cgc cca gtt gca aac cag acc tca ggc ggg ggc g \\
\hline $7 \mathrm{aL}$ & cgc ccc aag tgg ctc ctg acc tgg agt ctg ggc $\mathbf{g}$ \\
\hline $7 \mathrm{bU}$ & ccg gcg tgt tat ctc cta ggt tgg ctc tga ctg ccg g \\
\hline $8 \mathrm{aL}$ & cgc cgc acc get tct tgt cet get tgc ttc ggc $\mathbf{g}$ \\
\hline
\end{tabular}

Key to legend:

ID $=$ primer identifier

Sequence $=$ reverse primer and molecular beacon sequences. All molecular beacons contain 5' FAM (6-carboxyfluorescein) fluorophore and 3' methyl red quecher modifications. Selfcomplementary stem sequences are highlighted in bold. 
obtained from dilutions originating from one mutant cassette stock. Data were analyzed on the 7700 using the ROX-off setting, the ROX signal being used only to confirm template and reagent delivery. The cycle threshold was obtained for each well and the Cts of ARMS reactions correlated to the $\mathrm{Ct}$ of the corresponding control reaction to give a single well-specific data point. Data sets arising from the dilutions of wild-type and mutant templates were analyzed by linear regression to calculate the lines of best fit and $95 \%$ confidence intervals for each of the 80 tests on $1 \%$ mutant and wild-type templates. The resultant data were used to construct a diagnostic MS Excel macro that extracts the Cts of control reactions from an unknown sample and generates confidence limits around the expected ARMS reaction Cts for known wild-type and mutant harboring samples using the 80 reference data sets. For proof of principle with this prototype assay, the calculations assumed that the data obtained were representative of a continuous population whose $y$ variance is independent of $x$. The macro then simultaneously plots the observed and confidence limits for Cts from each of the 80 tests.

\section{Assay of Primary Tumor and Surgical Margins}

Real-time analyses were only performed on multiplex products from samples for which $>1000$ genomic copies had been input to the multiplex (multiplex functional on 1/1000 dilution of sample). Multiplex products from samples were diluted to add an estimated $10^{5}$ copies per well and subjected to real-time amplification as described. The 80 test-specific data points arising from each sample were analyzed with the diagnostic macro. Mutations were called where the ARMS Ct was earlier than both the lower confidence limit for a wild-type sample and the upper confidence limit for a sample harboring $1 \%$ mutant sequence. The results of the real-time assay were compared with those obtained using the FASAY/ phage plaque approach (3).

\section{Results}

p53 Multiplex

Three product bands of the expected sizes were clearly observed on agarose gel electrophoresis subsequent to multiplex amplification from all neat and 1/1000 dilutions of genomic samples. The ability to produce PCR products from $1 / 1000$ dilutions of sample demonstrates that sufficient copies of DNA were present in the neat sample to ensure (a) no false negatives are called due to insufficient DNA and (b) no false positives arise at the $1 \%$ level due to polymerase error in the initial round of amplification.

\section{Creation of Reference Data Sets}

Each of the 80 ARMS tests gave test $(y) \mathrm{Ct}$ data that were plotted against mean control $(x)$ Ct data from which lines of best fit and confidence intervals were calculated. Validation data from one of the 80 tests are illustrated in Figure 2. Each of the four control reactions were present in quadruplicate on every 96-well plate. To eliminate variability due to any preferential amplification of individual multiplex products, ARMS reaction Cts were always correlated with control PCR, which employed the same multiplex fragment as substrate. Data from cassettes harboring the same mutation, but of different lengths, independently created from different DNA stocks and primers, were indistinguishable (results not shown). The reference data sets permit the control Cts obtained from an unknown sample to be used to predict the ranges of expected Cts from each of the 80 ARMS tests if the sample were to be wildtype or contain $1 \%$ mutant sequence.

\section{Use of the Assay on Tumor and Surgical Margin Samples} Derived from Patients with Head and Neck Cancer

Real-time data obtained in the four control reactions (four replicates of each) on a clinical sample were used to generate $95 \%$ confidence intervals for

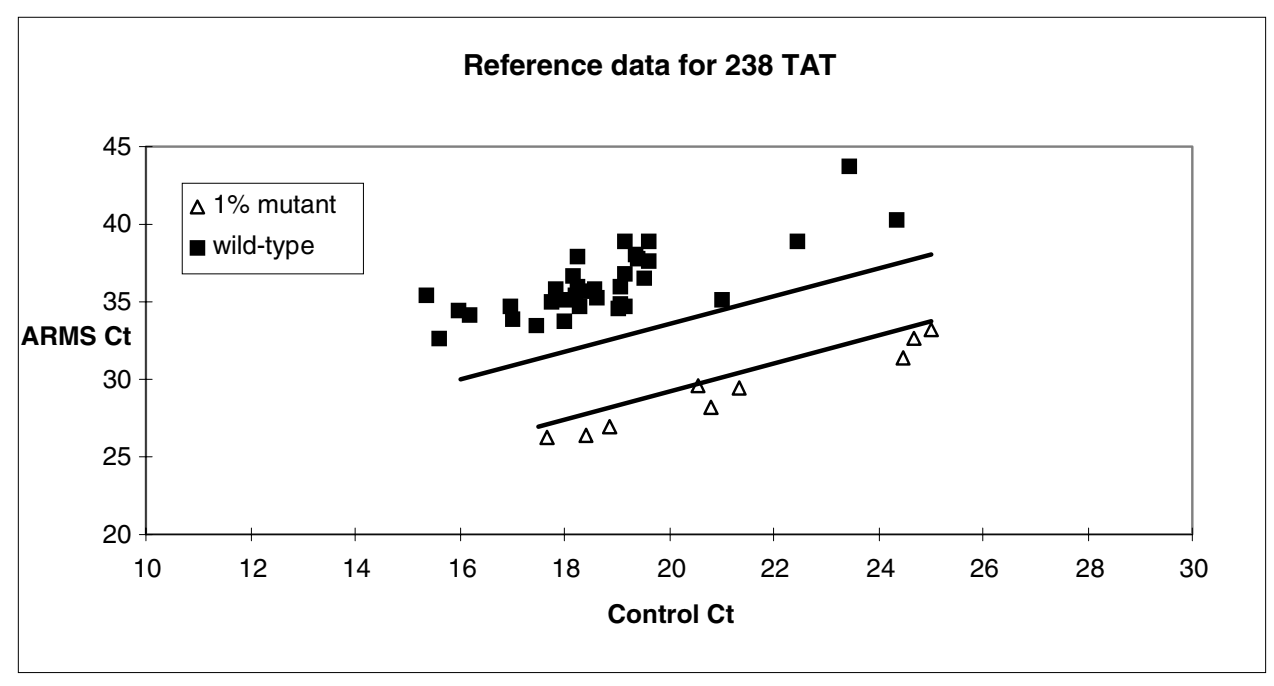

Fig. 2. Graph of example test data with confidence limits. Data were produced from wild-type DNA and samples harboring $1 \%$ mutant p53 sequences (238 tat). Each data point was acquired from a separate amplification reaction plate. The solid lines represent the lower and upper $95 \%$ confidence limits for the lines of best-fit for the wild-type and mutant data, respectively. For simplicity the data were assumed to be representative of a continuous population whose $y$ variance is independent of $x$. 
expected wild-type and $1 \%$ mutant $\mathrm{Ct}$ values for the 80 ARMS reactions run on the same 96-well plate. Sequence sites were diagnosed as wild-type unless the ARMS Ct was both excluded from the $95 \%$ wild-type confidence interval and included in the $1 \%$ mutant $95 \%$ confidence interval. In certain tests, where ARMS specificity was at its lowest, the 95\% confidence intervals for wild-type and $1 \%$ mutant samples overlapped slightly. In these instances, a small proportion of samples harboring p53 mutations as $1 \%$ of total p53 sequences go undetected. Where ARMS Cts are out of the wild-type range but not early enough to be included within the $1 \%$ confidence interval, the multiplex may harbor a mutation at less than $1 \%$ of total p53 sequences (see Discussion).

The graphical output from the use of the assay on DNA derived from a representative head and neck tumor and the matched surgical margins is illustrated in Figure 3. The tumor contains a high proportion of p53 sequences harboring the 175 cac mutation (well 20). This mutation is also present in the surgical margin at a much lower level. No other test $\mathrm{Ct}$ from the tumor or margin gives a value which is both out of the $\mathbf{9 7 . 5 \%}$ confidence intervals for normal sequence and within the confidence interval for mutant levels of $1 \%$ or greater. The results of the FASAY/phage plaque assay were disclosed subsequently and concurred with diagnosis by the realtime assay. The margin was estimated to contain $\sim 2 \%$ mutant sequence via the phage plaque assay.

\section{Discussion}

The major cause of morbidity and mortality following treatment for head and neck cancer is locoregional recurrence due to persistent or recurrent disease. Microscopic minimal residual cancer at the surgical margin has been shown to correlate with a high locoregional recurrence rate and decreased survival $(39,40)$. However, an ultrasensitive diagnostic test, which depends on finding the same p53 gene mutation in a primary tumor and the surrounding normal tissues considered to be tumor-free after conventional light microscopy, may allow detection of more cases with occult tumor at risk of local recurrence, such that these patients can be targeted to ensure that they receive early adjuvant treatment to eliminate any remaining malignant cells and improve their chance of cure $(2,3)$. However, this approach is a complex procedure with many sources for error. Self-ligation of the vector, due to partially degraded cDNA, is a significant problem. In addition, the protocol is very time consuming, such that incorporation of this technique, together with the p53 phage-plaque assay, is not practical in terms of developing a test that is quick, reliable, and can be automated to increase sample throughput and be used to influence decisions about patient treatment.

Many studies have used IHC detection of p53 as a proxy for detecting mutations in the $\mathrm{p} 53$ gene. The significance of such studies is open to debate; interpretation is subjective, about $25 \%$ of p 53 mutations do not result in protein overexpression, p53 protein levels may be elevated for other reasons, and the nature of the mutation present is not determined $(15,41)$. These difficulties may account for conflicting data concerning the relationship between immunoreactive p53 and prognosis or response to therapy. Direct DNA sequencing and hybridization techniques will only detect mutant $\mathrm{p} 53$ sequences present at levels greater than $25 \%$ of the total, unless they are applied to tumor-enriched samples. All tumor enrichment procedures involve a significant time/cost element and include visual selection (15), laser capture microdissection (42), and antibody binding (43). SSCP is compatible with scanning PCR products for mutations in a relatively routine, yet sensitive, manner and is currently the most widely used technique for genetic screening for p53 mutations $(29,30)$. However, SSCP is not quantitative and does not directly identify the mutation responsible for an observed band shift. What is required for routine application is a test that is quick, simple, and reliable, and that is not customized for each patient but is suitable for automation and amenable to quality control.

In the present study we describe a simple, quantitative, nucleic acid-based mutation detection system, capable of identifying specific $\mathrm{p} 53$ gene mutations against a background of tissue harboring wild-type alleles, likely to be very valuable in terms of prognostication and treatment planning for these patients. The 80-test panel described detects over $40 \%$ of p53 mutations reported in head and neck cancer (Table 1). The method relies entirely on the use of reference data sets to establish statistically validated "fingerprints" of the p53 status of tissues. The production of the reference datasets is relatively cost and labor intensive but, once generated, they provide a powerful diagnostic and quality control for data generated from clinical samples. Confidence in an accurate diagnosis of the composition of the multiplex products is provided by the presence of a Rox passive reference peak in each well, indicating the delivery of enzyme, template, and so on, as well as the conforming of the vast majority of ARMS test Cts to their expected wild-type values.

The assay produces highly reproducible data on any given multiplex product (data not shown) such that the performance of the multiplex is the critical step in the described procedure. It is imperative that the real-time assay data be interpreted in the knowledge of the minimum initial gene copy number input to the multiplex. If no bands are produced in the multiplex from a $1 / 1000$ dilution of tumor or margin DNA, then false negatives arise from the fact that the $1 \%$ detection limit is meaningless in the absence of $>100$ copies, and false positives may arise from the detection of a replication error from early in the amplification process. The multiplex is therefore necessary if samples are to be interrogated at large numbers of sites: for example, a test for 1000 mutations would require a minimum of $1000 \times$ 
$1000=1,000,000$ copies of the gene to add 1000 copies of the gene per test well. This corresponds to a minimum of $3 \mu \mathrm{g}$ of genomic material, which is unlikely to be forthcoming in many clinical situations. When bands are produced from the multiplex on dilute samples, similar false positives could arise if a simple non-wild-type analysis were performed, as certain ARMS tests can easily detect one mutant allele in the presence of 100,000 wild-type. It is therefore essential that, if data from a single, isolated run are to be interpreted, inclusion in the $1 \%$ data set is the criterion defining positivity, ensuring that even replication errors arising in the first round of amplification would not be accorded positive status.

The real-time ARMS assay successfully identified p53 mutations in clinical samples in blind trials. In the example shown, analysis of surgical margins from patients with head and neck cancer for the presence of tumor cells missed by conventional light microscopy detects the same mutation (175 CAC) in paired tumor and margin samples as independently detected in the phage-plaque assay. A further margin, in which an even lower level of mutant was detected by the yeast assay, was diagnosed as wildtype by the real-time ARMS assay. However, it is pertinent to note that the ARMS assay is highly quantitative, and thresholds may be set subsequent to data collection, such that clinical cut-offs can be optimized at a later date. The question of sufficient sensitivity for clinical utility can only be answered in a experiment in which the quantitative and objective data produced by such assays can be correlated to clinical outcome. The real-time assay described is amenable to use in such experiments because it is extremely easy to use, takes a day from sample to results, and requires less than 15 min of user intervention time to run; assuming stocks of multiplex primers and assay plates. We have also determined

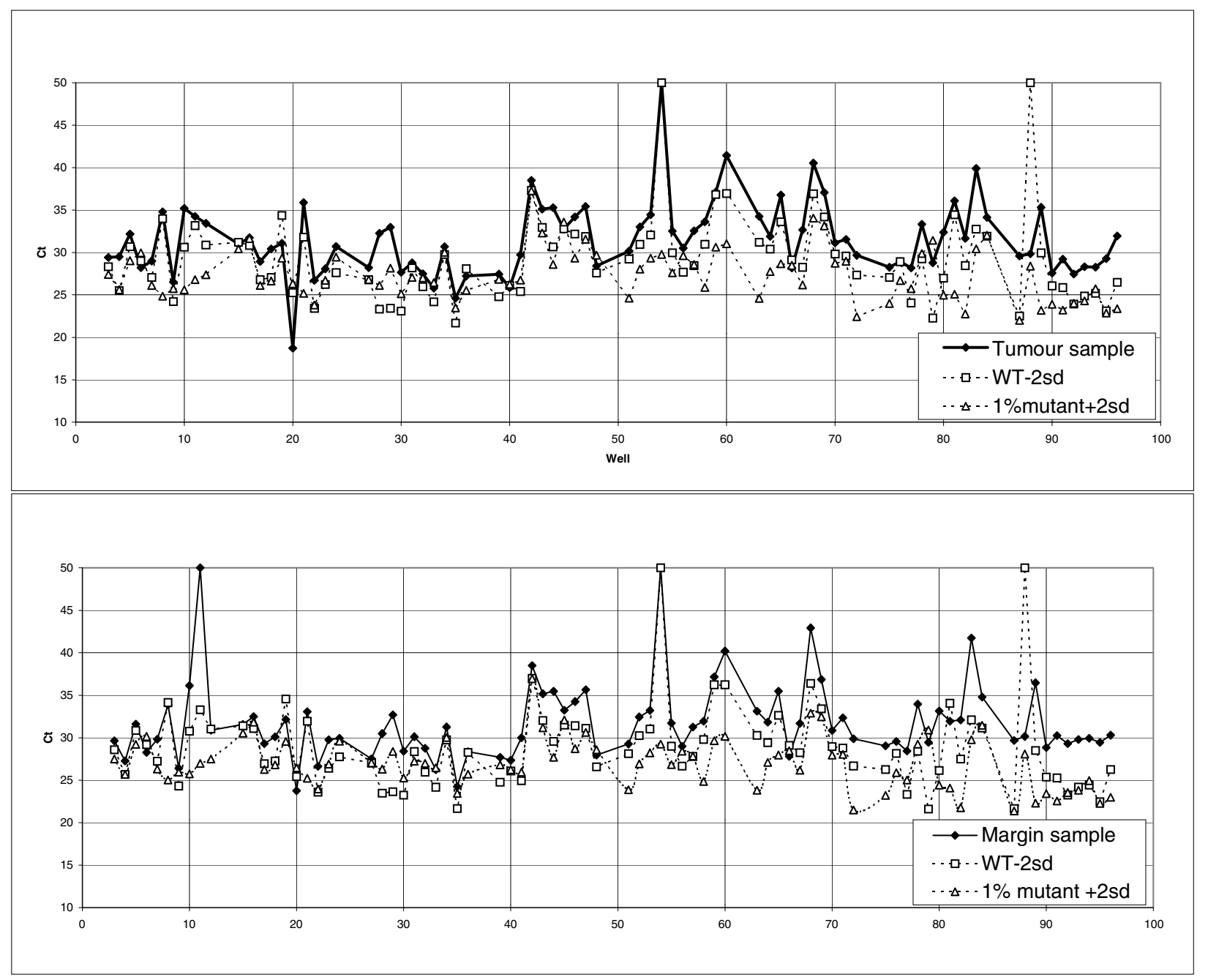

Fig. 3. Use of the assay on tumor and associated margin tissue. The Cts from the tumor and margin fall within the expected $97.5 \%$ confidence limits for all of the 80 tests except that in well 20, 175 cac. The mutation is clearly present as a larger proportion of the $\mathrm{p} 53$ sequences isolated from the tumor, but is also present within the tumor margin, where its presence implies a high probability of tumor tissue remaining within the patient after surgery. 
that real-time assay ready reagent plates can be stored frozen or dried without affecting the data produced (data not shown). In comparison with the yeast assay, the approach reduces the time taken to test a patient sample for residual tumor from 12 to 1 working day, and means that the tumor and each surgical margin can be screened for a 553 gene mutation to produce data sufficiently rapidly to be used to influence postoperative therapy. The closed-tube technology obviates the need for a gel, minimizes the risk of sample contamination, and ensures quality control. The cost of the raw materials used in the assay is around US $\$ 60.00$ per sample, but could be reduced considerably by miniaturization onto technology platforms currently reaching the market. The ARMS assay is sensitive, quick, simple, reliable, and robust enough to allow automation. In addition, as the assay uses closed-tube technology, the risk of crosscontamination of patient samples is minimized, a consideration paramount when translating molecular diagnostics from a research to a routine diagnostic setting.

The test panel described detects approximately $42 \%$ of p53 mutations reported in head and neck cancer and contains tests for the 80 most prevalent p53 mutations. Increasing the number of tests gives greater coverage of mutations. Table 4 shows that by increasing the number of tests to include the 240 most prevalent mutations, $75 \%$ coverage could be obtained. This approach is feasible using a suitable real-time PCR platform capable of handling 384well plates such as the Applied Biosystems 7900.

Table 4. Coverage of all p53 mutations obtained by increasing the number of tests included in the panel in order of their reported prevalence. Data obtained from the database maintained by $T$. Soussi, Laboratoire de Genotoxicologie des Tumeurs, Paris [35]

\section{Number of p53 \\ Mutations Tested}

\section{Percentage Coverage of all p53 Mutations Observed in Tumours}

\begin{tabular}{rr}
\hline 80 & 42 \\
90 & 48 \\
100 & 51 \\
120 & 56 \\
160 & 64 \\
240 & 75 \\
\hline
\end{tabular}

\section{Key to legend:}

Number of p53 mutations tested-Number of mutations included in a test panel.

Percentage coverage of all p53 mutations observed in tumoursMaximum percentage coverage of all $\mathrm{p} 53$ mutations that would be obtained by test panel.
In the present example, a mutation affecting the p53 gene is used as a "tumor fingerprint" to detect occult tumor. Such molecular analysis will play an increasingly important role in establishing whether margins are tumor-free or not. However, at this stage further studies are needed to clarify whether any existing and emerging treatments will be more efficacious if patients are stratified on the basis of p53 status. Based on the key position of the $\mathrm{p} 53$ protein in cell cycle regulation and current reports (44-48) it seems highly likely that incorporation of assays such as described here into routine clinical practice will enable information about the genetic aberrations associated with tumors to be used to plan treatment as well as providing an aid to diagnosis.

\section{Acknowledgments}

We thank Dr. Jayne Fox and Dr. Simon Clayton for helpful discussions. This work was supported by the AstraZeneca Strategic Development Fund.

\section{References}

1. Greenblatt MS, Bennet WP, Hollstein M, Harris CC. (1994) Mutations in the p53 tumour suppressor gene: clues to cancer etiology and molecular pathogenesis. Cancer Res. 54: 48554878.

2. Brennan JA, Mao L, Hruban RH, et al. (1995) Molecular assessment of histopathological staging in squamous-cell carcinoma of the head and neck. N. Engl. J. Med. 332: 429- 435.

3. Partridge M, Li S-R, Pateromichelakis S, et al. (2000) Detection of minimal residual cancer to investigate why oral tumours recur despite seemingly adequate treatment. Clin. Cancer Res. 6: 2718-2725.

4. Guinn BA, Mills KI. (1997) p53 Mutations, methylation and genomic instability in the progression of chronic myeloid leukaemia. Leuk. Lymphoma 26: 211-226.

5. Clahsen PC, van de Velde CJ, Duval C, et al. (1998) p53 protein accumulation and response to adjuvant chemotherapy in premenopausal women with node-negative early breast cancer. J. Clin. Oncol. 2: 470-479.

6. Iwao T, Hanada K, Tsuchida A, Hirata M, Eguchi N, Kajiyama G. (1998) The establishment of a preoperative diagnosis of pancreatic carcinoma using cell specimens from pancreatic duct brushing with special attention to 553 mutations. Cancer 82: 1487-1494.

7. Weller M. Predicting response to cancer chemotherapy: the role of p53. (1998) Cell Tissue Res. 292: 435-445.

8. Herr HW, Bajorin DF, Scher HI, Cordon-Cardo C, Reuter VE. (1999) Can p53 help select patients with invasive bladder cancer for bladder preservation? J. Urol. 161: 20-23.

9. Aas T, Borresen AL, Geisler S, et al. (1996) Specific p53 mutations are associated with de novo resistance to doxorubicin in breast cancer patients. Nat. Med. 2: 811-814.

10. Berns EM, van Staveren IL, Look MP, Smid M, Klijn JG, Foekens JA. (1988) Mutations in residues of TP53 that directly contact DNA predict poor outcome in human primary breast cancer. Br. J. Cancer 77: 1130-1136.

11. Burke HB, Hoang A, Iglehart JD, Marks JR. (1998) Predicting response to adjuvant and radiation therapy in patients with early stage breast carcinoma. Cancer 82: 874-877.

12. Iniesta P, Vega FJ, Caldes T, et al. (1998) p53 exon 7 mutations as a predictor of poor prognosis in patients with colorectal cancer. Cancer Lett. 130: 153-160.

13. Børresen-Dale A-L. (1997)Subgroups of p53 mutations may predict the clinical behaviour of cancers in the breast and colon and contribute to therapy response. In Klijn JGM (ed). ESO Scientific Updates, Vol 1. Prognostic and Predictive Value of $p 53$. 
14. Erber R, Conradt C, Homann N, et al. (1998) TP53 DNA contact mutations are selectively associated with allelic loss and have a strong clinical impact in head and neck cancer. Oncogene 16: 1671-1679.

15. Berns EM, Foekens JA, Vossen R, et al. (2000) Complete sequencing of TP53 predicts poor response to systemic therapy of advanced breast cancer. Cancer Res. 60: 2155-2162.

16. Temam S, Flahault A, Périé S, et al. (2000) p53 gene status as a predictor of tumor response to induction chemotherapy of patients with locoregionally advanced squamous cell carcinomas of the head and neck. J. Clin. Oncol. 18: 385-394.

17. Ogawa Y, Nishioka A, Hamada N, et al. (1998) Changes of mutant-type p53 expression in squamous cell carcinoma of the head and neck during radiation therapy and its clinical significance: comparison of an immunohistochemical method and PCR-SSCP assay. Oncol. Rep. 5: 1053-1059.

18. *Cabelguenne A, Blons $\mathrm{H}$, de Waziers I, et al. (2000) p53 alterations predict tumor response to neoadjuvant chemotherapy in head and neck squamous cell carcinoma: a prospective series. J. Clin. Oncol. 18: 1465-1473.

19. Bradford CR, Zhu S, Wolf GT, et al. (1995) Overexpression of p53 predicts organ preservation using induction chemotherapy and radiation in patients with advanced laryngeal cancer. Otolaryngol. Head Neck Surg. 113: 408-412.

20. Obata A, Eura M, Sasaki J, et al. (2000) Clinical significance of p53 functional loss in squamous cell carcinoma of the oropharynx. Int. J. Cancer (Pred. Oncol.) 89: 187-193.

21. Saunders ME, MacKenzie R, Shipman R, Fransen E, Gilbert R, Jordan RCK. (1999) Patterns of p53 gene mutations in head and neck cancer: full-length gene sequencing and results of primary radiotherapy. Clin. Cancer Res. 5: 2455-2463.

22. Awwad S, Jaros E, Somes J and Lunec J. (1996) p53 overexpression in head and neck carcinoma and radiotherapy results. Int. J. Radiation Oncol. Biol. Phys. 34: 323-332.

23. Brown JM, Wouters BG. (1999) Apoptosis, p53 and tumor cell sensitivity to anticancer agents. Cancer Res. 59: 1391-1399.

24. Partridge M. (1999) Head and neck cancer and precancer: can we use molecular genetics to make better predictions? Ann. R. Coll. Surg. Engl. 81: 1-11.

25. Partridge M. (2000) Current status of genetics for prediction, prognosis and gene therapy. Curr. Opin. Otolaryngol. 8: 69-80.

26. Barbareschi M. (1996) Prognostic value of the immunohistochemical expression of $\mathrm{p} 53$ in breast carcinomas. A review of the literature involving over 9000 patients. Applied Immunohistochemistry 4: 106-116.

27. Flaman JM, Frebourg T, Moreau V, et al. (1995) A simple p53 functional assay for screening cell lines, blood and tumours. Proc. Natl. Acad. Sci. U.S.A. 92: 3963-3967.

28. Ahrendt SA, Halachmi S, Chow JT, et al. (1999) Rapid p53 Sequence Analysis in Primary Lung Cancer Using an Oligonucleotide Probe Array. Proc. Nat. Acad. Sci. U.S.A. 96: 7382-7387.

29. Kutach LS, Bolshakov S, Ananthaswamy HN. (1999) Detection of mutations and polymorphisms in the p53 tumor suppressor gene by single-strand conformation polymorphism analysis. Electrophoresis 20: 1204-1210.

30. Iacopetta B, Elsaleh H, Grieu F, Joseph D, Sterrett G, Robbins P. (2000) Routine analysis of p53 mutation in clinical breast tumour specimens using fluorescence-based polymerase chain reaction and single strand conformation polymorphism. Diagn. Mol. Pathol. 9: 20-25.
31. Suwinski R, Taylor JMG, Withers HR. (1998) Rapid growth of microscopic rectal cancer as a determinant of response to preoperative radiation therapy. Int. J. Radiat. Oncol. Biol. Phys. 42: 943-951.

32. Newton CR, Graham A, Hepinstall LE, Powell SJ, Summers C, Kalsheker N. (1989) Analysis of any point mutation in DNA. The amplification refractory mutation system (ARMS). Nucleic Acids Res. 17: 2503-2515.

33. Heid CA, Stevens J, Livak KJ, Williams PM. (1996) Real time quantitative PCR. Genome Res. 6: 986-994.

34. Tyagi S, Bratu, DP, Kramer FR. (1998) Multicolor molecular beacons for allele discrimination. Nature Biotech. 16: 49-53.

35. Available from URL: http://perso.curie.fr/Thierry.Soussi/ seqp53/Gene-p53.html. Accessed throughout 1999.

36. Ferrie RM, Schwarz MJ, Robertson NH, et al. (1992) Development, multiplexing, and application of ARMS tests for common mutations in the CFTR gene. Am. J. Hum. Genet. 51: 251-262.

37. Higuchi R, Krummel B, Saiki RK. (1988) A general method of in vitro preparation and specific mutagenesis of DNA fragments: a study of protein and DNA interactions. Nucleic Acids Res. 16: 7351-7367.

38. Available from URL: http://www.ibc.wustl.edu/ zuker. Accessed throughout 1999.

39. Cook JA, Jones AS, Phillips DE, Soler Lluch E. (1993) Implications of tumour in resection margins following surgical treatment for squamous cell carcinoma of the head and neck. Clin. Otolaryngol. 18: 37-41.

40. Jones AS, Bin Hanafi Z, Nadapalan V, Roland NJ, Kinsella A, Helliwell TR. (1996) Do positive resection margins after ablative surgery for head and neck cancer adversely affect prognosis? A study of 352 patients with recurrent carcinoma following radiotherapy treated by salvage surgery. Br. J. Cancer 74: 128-132.

41. Leitner T, Halapi E, Scarlatti G, et al. (1993) Analysis of heterogeneous viral populations by direct DNA sequencing. Biotechniques 15: 120-126.

42. Simone NL, Bonner RF, Gillespie JW, Emmert-Buck MR, Liotta LA. (1998) Laser capture microdissection: opening the microscopic frontier to molecular analysis. Trends Genet. 14: 272-276.

43. Masson D, Denis MG, Lustenberger P. (2000) Limitations of CD44v6 amplification for the detection of tumour cells in the blood of colorectal cancer patients. Br. J. Cancer 82: 1283-1289.

44. Lee JG. (1974) Detection of residual carcinoma of the oral cavity, oropharynx, hypopharynx and larynx: a study of surgical margins. Trans. Am. Acad. Ophthalmol. Otolaryngol. 78: 49-53.

45. Jesse RH, Sugarbaker EV. (1976) Squamous cell carcinoma of the oropharynx: why we fail. Am. J. Surg. 132: 435-438.

46. Scholl P, Byers RM, Batsakis JG, Wolf P, Santini H. (1986) Microscopic cut-through of cancer in the surgical treatment of squamous carcinoma of the tongue: prognostic and therapeutic implications. Am. J. Surg. 152: 354-360.

47. Zieske LA, Johnson JT, Myers EN, Thearle PB. (1986) Squamous cell carcinoma with positive margins: surgery and postoperative irradiation. Arch. Otolaryngol. Head Neck Surg. 112: 863-866.

48. Khuri FR, Nemunaitis J, Ganly I, et al. A controlled trial of intratumoral ONYX-015, a selectively-replicating adenovirus, in combination with cisplatin and 5-fluorouracil in patients with recurrent head and neck cancer. Nat. Med. 6: 879-885. 